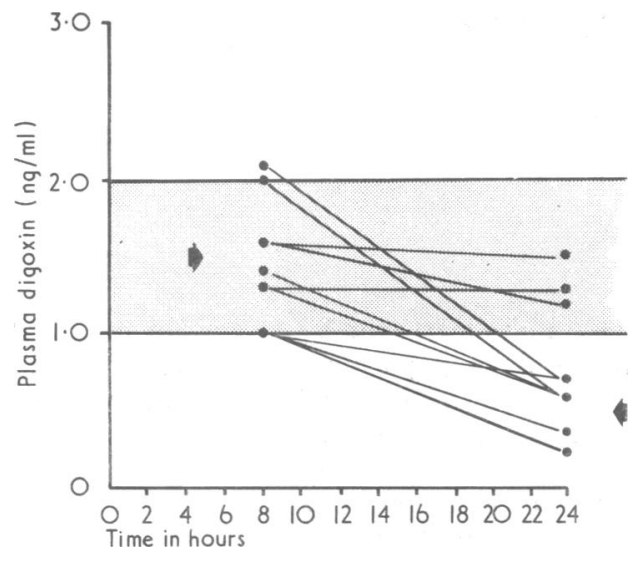

FIG. 2-Fall in digoxin concentrations in $\mathbf{1 0}$ children after a single daily dose. Shaded area represents therapeutic adult range. Arrows indicate mean values.

below the optimum adult range of 1-2 $\mathrm{ng}$ at the end of the 24hour period (fig. 2).

\section{Discussion}

Measurement of plasma digoxin concentrations has proved valuable in determining the therapeutic dose of the drug in adults, and provides a means of studying its pharmacology (Smith et al., 1969; Chamberlain et al., 1970; Beller et al., 1971).

The rationale behind recommendations for digoxin dosages in infants and children has never been clarified. Levine (1962) attempted to determine clinically the maximum safe digitalizing dose for infants and children, and arrived at a dosage similar to the recommended schedule in this study.

Infants in the first month of life receiving half the daily dose given to older infants produce similar plasma concentrations. This may be because of reduced renal clearance in the neonatal period. The doses for all age groups are substantially higher on a mg per kg body weight basis than those for adults; our data show that identical plasma concentrations are produced by infants receiving a maintenance dose of $0.02 \mathrm{mg} / \mathrm{kg}$ body weight and adults receiving $0.007 \mathrm{mg} / \mathrm{kg}$ body weight. This difference persists when doses are calculated on body surface area, although it is less pronounced. It is thought that children are more tolerant to digitalis glycosides, but not infrequently intoxication has occurred from over-medication. We have not attempted to show if larger doses cause toxicity, and it may be that the infant's myocardium will tolerate higher plasma levels than those produced by our dose schedules. Failure to secure adequate clinical control of cardiac failure in an infant may suggest a need to increase the digoxin dosage, but it is our impression that an increase in dosage above that of our schedule does not lead to an enhanced therapeutic effect. This impression is supported by recent studies (Levy et al., 1972) showing that a maximal inotropic effect occurs after a single small dose.

By measuring plasma digoxin levels after a single daily dose we have shown that in seven cases out of 10 the therapeutic adult range of 1-2 ng is not maintained 24 hours after the dose. It is, therefore, desirable to follow the usual practice of twice daily dosage.

The paediatric dose schedule presented here produces plasma digoxin levels comparable to those in adults with normal renal function receiving $0.5 \mathrm{mg}$ daily to control atrial fibrillation. Our clinical observations also support our belief that this dosage regimen is effective and safe.

We thank Dr. D. A. Chamberlain for his help in the preparation of this paper. We are grateful to Dr. T. P. Mann, Dr. F. W. Nash, and Dr. G. W. Hatcher, of the Royal Alexandra Hospital, Brighton, and Professor J. F. Goodwin, of the Postgraduate Medical School, London, for permission to study patients under their care.

Dr. J. E. Cree was financed by the Royal Alexandra Hospital Research Fund, and Dr. D. J. Coltart is the Mary Scharlieb Research Scholar of the University of London.

Requests for reprints should be addressed to: Dr. J. E. Cree, Royal Alexandra Hospital for Sick Children, Dyke Road, Brighton BN1 3JN.

\section{References}

Beller, G. A., Smith, T. W., Abelmann, W. H., Haber, E., and Hood, W. B. Jun. (1971). New England fournal of Medicine, 284, 989. Chamberlain, D. A., White, R. J., Howard, M. R., and Smith, T. W. (1970). British Medical fournal, 3, 429.

Levine, O. R., and Blumenthal, S. (1962). Pediatrics, 29, 18.

Levy, A. M., Leaman, D. M., and Hansom, J. S. (1972). Circulation, 46, 816. Nadas, A. S. (1966). Paediatric Cardiology, 2nd edn., p. 783. London, Saunders.

Smith, T. W., Butler, V. P., and Haber, E. (1969). New England fournal of Medicine, 281, 1212 .

Taylor, J. F. N. (1971). British fournal of Hospital Medicine, 6, 450.

\title{
Rubella Epidemic in a Maternity Unit
}

\section{STUART CARNE, C. J. DEWHURST, ROSALINDE HURLEY}

British Medical fournal, 1973, 1, 444-446

\section{Summary}

Four cases of rubella, the last confirmed by laboratory tests, occurred among the nurses of a large obstetric unit. Immediate measures were taken to prevent the spread of the infection to pregnant patients and staff, and none was in fact infected.

Queen Charlotte's Maternity Hospital and the Institute of Obstetrics and Gynaecology, University of London, London W6 0XG STUART CARNE, M.B., F.R.C.G.P., General Practitioner to the Staff C. J. DEWHURST, F.R.c.S., F.R.c.o.G., Professor of Obstetrics and Gynaecology
ROSALINDE HURLEY, M.D., M.R.C.PATH., Consultant Microbiologist
Persons susceptible to rubella and who become infected may pose a real danger to women in early stages of gestation during the incubation period. We recommend that the immune status of medical and nursing personnel working in obstetric departments should be ascertained serologically, and that rubella vaccine should be offered to those who are susceptible.

\section{Introduction}

About $20 \%$ of women of childbearing age in southern England are susceptible to rubella (Public Health Laboratory Service Working Party, 1970; Beazley et al., 1971). Studies on immunity to rubella have been made on women in closed 
communities (Dudgeon et al., 1969a), and a high proportion (about $35 \%$ ) of junior nursing staff in one hospital were known to be susceptible to rubella (Dudgeon et al., 1969b). There is, therefore, no evidence that unvaccinated nurses are less susceptible to rubella than are other young women in the community, although it might appear probable that more senior nurses, as well as doctors, are likely to be immune because of the greater probability of occupational exposure to the disease.

This is an account of an epidemic of rubella which was confined to the nurses of a maternity unit; of the measures that were taken to ensure that the disease had not and did not spread to susceptible pregnant women; and of proposals for the prevention of similar occurrences.

\section{The Cases}

On Friday, 5 May, one of us was informed that three cases of clinically diagnosed rubella had occurred among the nursing staff of our maternity unit. So far as could be ascertained, the rashes had appeared on 21 April, 3 May, and 4 May. Rubella had been diagnosed by the nurses' own general practitioners, all were being nursed at home, and no specimens were available for laboratory confirmation of the disease. Since no other cases had arisen within the hospital community, and none of the inpatients had had rubella, the ultimate source was thought to be exogenous to the hospital. The affected nurses had worked in the labour ward, the premature baby unit, and the outpatients' department, but all had had opportunity to mix freely with other staff employed throughout the hospital. Since many of these are trained midwives and doctors whose immune status with respect to rubella was at that time unknown, and who come into close contact with women in early pregnancy, we decided to take prompt measures to ascertain that seroconversion had not taken place in pregnant women under our care, and to determine the immune status of the staff, so that we might recognize those among us who were at risk of rubella.

On 16 May a rash appeared in a fourth nurse who had been taken off duty in one of the lying-in wards because of malaise three days before. She was isolated in the nurses' sick bay. Rubella was confirmed serologically in this girl, and a more stringent policy to prevent the possibility of spread to the patients was adopted. No further cases occurred.

\section{Epidemiological Investigations}

Sera stored from booking tests from all patients under 16 weeks' gestation during the relevant period, who had either been admitted to the hospital or seen in the antenatal clinic, were screened at $1 / 32$ by the rubella H.A.I. test. Those who were negative were regarded as susceptible and the test was repeated to detect seroconversion indicating recent contraction of rubella, using the rubella H.A.I. test (Beazley et al., 1971) and titrating up to at least $1 / 512$. Altogether, 159 sera were examined; 151 were seropositive on booking. Seroconversion did not occur in any of the seronegative women who were retested.

Sera were also screened from those medical and nursing staff whose rubella immune status had not previously been determined. Twenty-three of the 204 examined were negative at $1 / 32$. (Only one of the medical staff was seronegative.) Cendehill vaccine was offered and accepted by all those staff who were susceptible, with the exception of one of the senior members of the nursing staff, who was due to retire and had no direct contact with patients.

After the occurrence of the fourth case, the only one in which the diagnosis was confirmed by the laboratory, no patient likely to be under 16 weeks' pregnancy was admitted to the hospital or booked in the antenatal clinics. Those whose visits could not be deferred were seen at the Chelsea Hospital for Women, and patients in later stages of gestation were examined by those who were known to be immune to rubella where possible. The clinics were not reopened to women in early pregnancy until about a month after the appearance of the last rash.

As soon as notification of the first three cases of rubella had been received pregnant hospital staff were tested serologically and the tests were repeated about two weeks later to detect any antibody rise that might indicate infection or reinfection. None developed rubella. They were given leave of absence until one month after the appearance of the last rash, irrespective of the length of gestation of their pregnancies. Three of the four pregnant staff remained off duty, and one chose to continue her work in an isolated part of the hospital, refraining from contact with medical and nursing staff and patients.

The measures taken to detect susceptible persons and to protect the pregnant and non-pregnant population are summarized below.

(1) Patients under 16 weeks' pregnancy were not admitted or booked in the antenatal clinics after the occurrence of the fourth (proved) case. The clinics were reopened fully one month after the appearance of the last rash.

(2) Booking blood from all patients under 16 weeks' pregnancy over the relevant period was tested serologically and the test was repeated in seronegative patients.

(3) The immune status of all medical and nursing personnel was established, and Cendehill vaccine was offered to the susceptible persons.

(4) Other patients were examined and cared for by medical and nursing staff known to be immune to rubella where possible.

(5) Pregnant staff were retested if susceptible, and were given leave of absence until one month after the appearance of the last rash.

\section{Discussion}

The incubation period of rubella from the date of known exposure to the rash is usually 17 to 18 days, although extremes of 12-22 days have been recorded. Patients with rubella are infectious during the last week of the incubation period and virus can be recovered from the nasopharynx as early as 13 days before the rash (Sever et al., 1963) and up to 14 days after the rash, although viraemia seldom persists for more than a day after the rash (Dudgeon ,1967; Green et al., 1964). Since the first rash reported to us occurred on 21 April, we deemed it wise, and it was administratively easier, to suppose that patients and staff may have been exposed to rubella since the beginning of April. Therefore we tested the sera of all women in early pregnancy examined in our unit since 1 April. The rashes of the second and third cases occurred on 3 and 4 May. It is possible that both these girls contracted rubella from the first case, since the onset of their symptoms falls within the reported incubation period, but it is also possible that all three were exposed to a common source. The rash of the fourth case occurred on 16 May; this girl could have contracted the disease from case one or even from cases two or three, since this would fall within the incubation period. Thus, it was likely that case to case transmission was occurring in our hospital community.

Since there is no means of telling for certain who, of susceptible persons, may be incubating the disease, and since both reinfection and subclinical infection may occur, we decided to defer the visits of women likely to be under 16 weeks pregnant for a period approximating to twice the normal incubation period of the disease. The last patient with rubella had been isolated on $12 \mathrm{May}$, and the clinics were reopened on 14 June. 
No further cases of rubella occurred, and there was no clinical or serological evidence that any women in early pregnancy under our care at the time of the epidemic contracted the disease.

Immune globulin was not given to susceptible personnel for fear of masking the continuation of the epidemic, but rubella vaccine was offered and was given. The side effects of the vaccine-rash, mild arthralgia, or constitutional disturbance-were not stressed, but female recipients were advised to take positive steps to avoid pregnancy in the six weeks after vaccination.

Although the percentage of susceptible people was lower than previously recorded in both patients and staff, the difference was not statistically significant when compared with the results of previous studies in the same hospital. The danger that nurses and doctors susceptible to rubella may pose to patients in early pregnancy in the course of an epidemic was made apparent to us by this episode. Contact in antenatal clinics is very close and could have disastrous consequences for the fetus if the medical attendant is incubating rubella and is infectious. It has been our policy to protect susceptible nurses working in our unit by offering Cendehill vaccine. It seems advisable that the immune status of doctors, nurses, or other attendants who may come into close contact with patients in early pregnancy, should be ascertained serologically, and that seronegative persons should be encouraged to accept vaccination in the interests of the patients. Such will be our policy henceforth.

We are grateful to J. Bebbington, who performed the serological tests.

\section{References}

Beazley, J. M., Hurley, Rosalinde, Middlebrook, Celia, and Rumpus, M. F. (1971). British fournal of Preventive and Social Medicine, 25, 140.

Dudgeon, J. A. (1967). In Modern Trends in Medical Virology, ed. R. B. Heath and A W aterson, 111 .

Dudgeon, J. A., Marshall, W. C., Peckham, Catherine S., and Hawkins, G. T. (1969a). British Medical' Fournal, 1, 271.

Dudgeon, J. A., Marshall, W. C., and Peckham, Catherine S. (1969b). American fournal of Diseases of Childhood, 118, 237.

Green, R. H., Balsamo, M. R., Giles, J. P., Krugman, S., and Mirick, G. S. (1964). Transactions of the Association of American Physicians, 77, 118. Public Health Laboratory Working Service Party on Rubella (1970). British Medical Fournal, 2, 497.

Sever, J. L., Brody, J. A., Schiff, G. M., McAlister, R., and Cutting, R. (1963). Fournal of the American Medical Association, 191, 624.

\title{
Effect of Metoclopramide on Gastric Emptying during Labour
}

\author{
F. A. HOWARD, D. S. SHARP
}

British Medical fournal, 1973, 1, 446-448

\begin{abstract}
Summary
The effect of a single dose of metoclopramide (Maxolon) on the gastric emptying rate of a liquid test meal was measured in a group of parturient women by a doublesampling dye-dilution technique. The subjects were given either metoclopramide $10 \mathrm{mg}$ or sterile water by intramuscular injection on a double-blind random basis at the start of the test. The gastric emptying rate was significantly increased in those women who had received the metoclopramide as compared with those who had received the placebo.
\end{abstract}

\section{Introduction}

Mendelson (1946) first reported the development of acute pulmonary oedema after the inhalation of vomitus in women in labour undergoing emergency general anaesthesia. Since then successive reports on maternal mortality have drawn attention to this syndrome. In the Confidential Enquiries into Maternal Deaths in England and Wales covering the years 1967-9 (Department of Health and Social Security, 1972) a total of 451 maternal deaths were considered. Of these, 50 were associated with general anaesthesia and at least half of these were caused by the inhalation of regurgitated gastric contents. These figures were

Department of Obstetrics and Gynaecology, University Hospital of South Manchester, Manchester M20 8LR

F. A. HOWARD, M.B., M.R.C.o.G., Senior Registrar

D. S. SHARP, M.B., M.R.C.o.G., Registrar remarkably and disappointingly similar to those contained in the previous two triennial reports on maternal mortality.

Obstetricians have long been aware of the hazard constituted by vomiting associated with emergency general anaesthesia in women during labour. In such patients the vomitus may contain the remnants of food ingested many hours previously, and it is largely as a result of such clinical experience that it has been thought that the gastric emptying rate is substantially decreased in these women. Such studies that have been reported support this belief. Hansen (1937) attempted to measure the gastric emptying rate by administering a fluid test meal containing methylene blue and then aspirating samples from the stomach until such time as there was no further trace of the dye in the aspirate. In this way he showed that the gastric emptying time of pregnant women was about double that of non-pregnant women. Davison et al. (1970) compared the gastric emptying times for a fluid test meal in normal, non-pregnant women and women in late pregnancy. While calculations of the half life of gastric emptying showed no significant difference between the two groups the volumes of fluid remaining in the stomach after 30 minutes were significantly greater in the pregnancy group.

Metoclopramide is a chlorbenzamide derivative which uas been shown to inhibit vomiting induced centrally by apomorphine (Laville and Margarit, 1964) and to accelerate gastric emptying by a direct effect on the autonomic nuclei (Jacobie and Brodie, 1967). Eisner (1968) showed by in-vitro studies that metoclopramide sensitizes gastric smooth muscle fibres to the effect of acetylcholine, thus potentiating the effect of the latter. There are many reports of the antiemetic properties of metoclopramide. Clark and Storrs (1969) showed a reduced incidence of postoperative vomiting in patients treated with metoclopramide, and Johnson (1971) demonstrated its value in the management of flatulent dyspepsia. Several workers have reported favourably on its use in treating vomiting associated with early pregnancy, 